Noam R. Shabtai, Gottfried Behler, Michael Vorländer, Stefan Weinzierl

\title{
Generation and analysis of an acoustic radiation pattern database for forty-one musical instruments
}

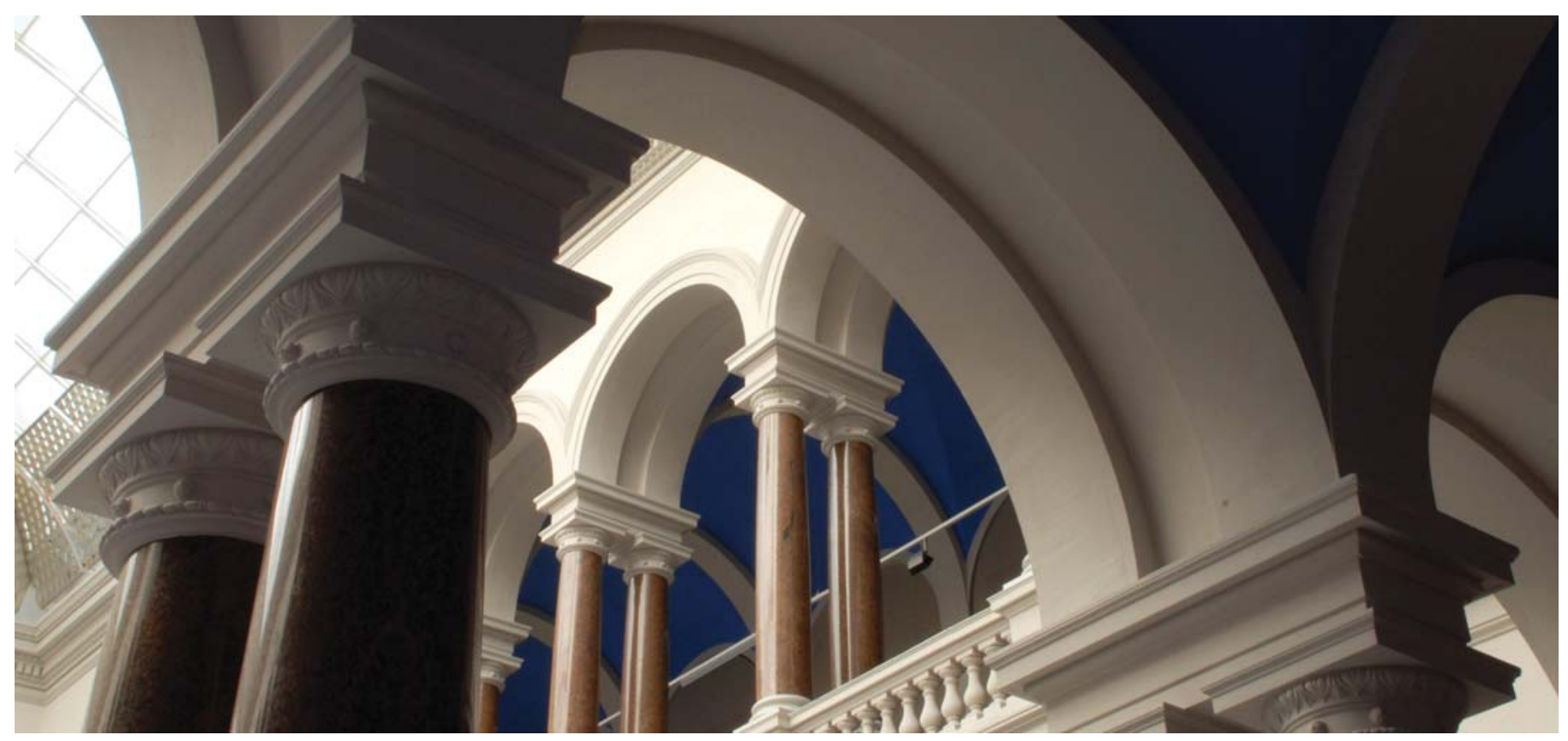

The following article appeared in

Shabtai, N. R., Behler, G., Vorländer, M., \& Weinzierl, S. (2017). Generation and analysis of an acoustic radiation pattern database for forty-one musical instruments. The Journal of the Acoustical Society of America, 141(2), 1246-1256.

and may be found at

https://doi.org/10.1121/1.4976071

\section{Terms of Use}

Copyright (2017) Acoustical Society of America. This article may be downloaded for personal use only. Any other use requires prior permission of the author and the Acoustical Society of 


\title{
Generation and analysis of an acoustic radiation pattern database for forty-one musical instruments
}

\author{
Noam R. Shabtai, ${ }^{\text {a) }}$ Gottfried Behler, Michael Vorländer \\ Institute of Technical Acoustics, RWTH Aachen University, Kopernikusstr. 5, 52074 Aachen, Germany \\ Stefan Weinzierl
}

Audio Communication Group, Technical University Berlin, Einsteinufer 17, 10587 Berlin, Germany

\begin{abstract}
A database of acoustic radiation patterns was recorded, modeled, and analyzed for 41 modern or authentic orchestral musical instruments. The generation of this database included recordings of each instrument over the entire chromatic tone range in an anechoic chamber using a surrounding spherical microphone array. Acoustic source centering was applied in order to align the acoustic center of the sound source to the physical center of the microphone array. The acoustic radiation pattern is generated in the spherical harmonics domain at each harmonic partial of each played tone. An analysis of the acoustic radiation pattern complexity has been performed in terms of the number of excitation points using the centering algorithm. The database can be used both for studying the radiation of musical instruments itself, as well as for the implementation of radiation patterns in room acoustical simulations and auralization in order to obtain a spatial excitation of the room closer to reality.
\end{abstract}

\section{MOTIVATION}

Room-acoustics simulations usually provide source-toreceiver room impulse responses (RIRs). The first models were developed using the image-source method for shoe-box room models. ${ }^{1,2}$ Great efforts have been made since then in simulating the receiver characteristics, mainly by incorporating the head related transfer functions, ${ }^{3}$ in order to generate binaural room impulse responses. ${ }^{4}$ Other efforts have been made to generate RIRs of more complex environments, combining the image-source for the early reflections with ray tracing for the late reflections, as first presented in 1989 by Vorländer, ${ }^{5}$ later known as the Real-time framework for the Auralization of interactive Virtual ENvironments (RAVEN) project. $^{6}$ The ODEON system is also built on this hybrid method. ${ }^{7}$

Although methods are available that allow incorporating the spatial radiation of the source in addition to the directivity of the receiver in room simulations, ${ }^{8}$ musical instrument radiation patterns are typically not integrated into such systems. The reason is probably that the information available on the directivity of real musical instruments was relatively limited to a small amount of musical instruments using relatively low spatial resolution until approximately the year of 2009.

Several attempts have been made to measure the radiation pattern of musical instruments, perhaps first by Meyer in the $1970 \mathrm{~s},{ }^{9}$ followed by measurements using surrounding spherical microphone array of 12 microphones, ${ }^{10}$ and using 13 microphones in two surrounding circular arrays. ${ }^{11}$ The radiation pattern of singers has also been measured. ${ }^{12} \mathrm{~A}$ more extensive analysis was performed for 8 instruments using 64 microphones $^{13}$ and for 22 instruments using 22

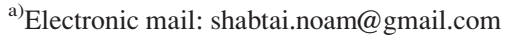

microphones. ${ }^{14}$ The most comprehensive measurement series has been performed in the Technical University of Berlin (TU Berlin) for 41 modern and authentic musical instruments, i.e., of different historical periods of instrument making, using a spherical array of 32 microphones. ${ }^{15}$ More advanced measurements are performed automatically without a human player so as to repeat the signals and turning the instruments in order to increase the spatial resolution without increasing the number of microphones. These automatic measurements can be performed using an artificial mouth using brass instruments, ${ }^{16}$ or for string instruments, using the Lorenz force principle by injecting an electric current to one of the strings under a magnetic field. ${ }^{17}$

A specific problem in the modeling of the radiation pattern of musical instruments is a definition and estimation of an acoustical center. ${ }^{18,19}$ Radiated sound from musical instruments with their spatially extended and note-dependent vibration patterns often does not emerge in the center of the microphone array that is surrounding the musical instrument. Furthermore, the difference in arrival times from the source results in phase shifts at the microphone positions. In case there is more than one contributing source, interference may occur, resulting in an even higher complexity of the radiation pattern. Furthermore, in order to represent the radiation pattern in this case, it is still required to define a unique acoustic center. Acoustic source centering is probably the most mathematically demanding stage in the generation of this database.

This work represents the generation of a mathematically formulated database of a radiation pattern from a set of 41 musical instruments, based on the measurements performed in TU Berlin in 2009 using a surrounding spherical array of 32 microphones. ${ }^{15}$ A quick overview of the system is shown in Fig. 1. Section II describes the calibration of the electrical 


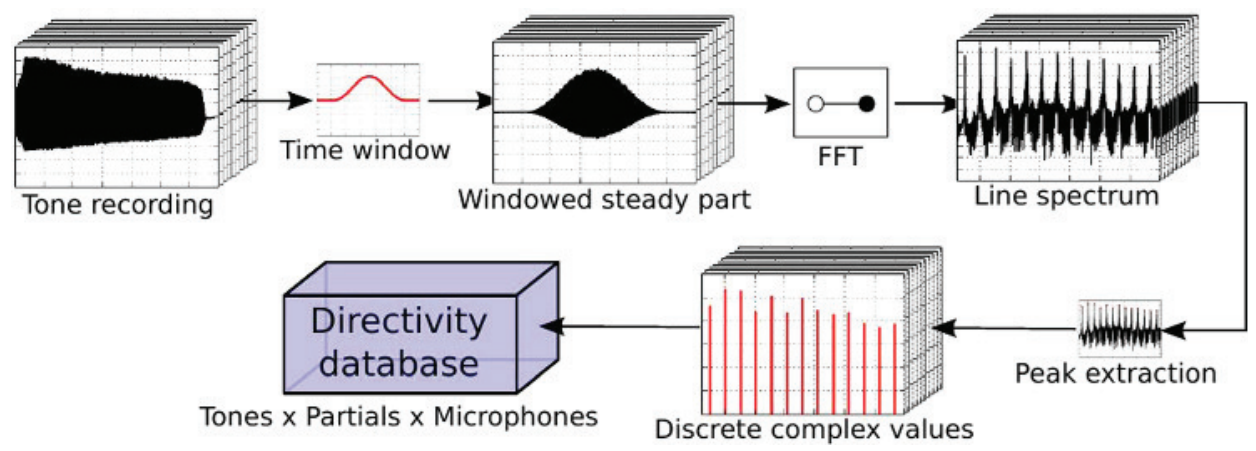

FIG. 1. (Color online) Overview of the radiation pattern recording scheme (Ref. 21).

channels and the measurement of the frequency response of the microphones. ${ }^{20}$ Section III describes the recording session in an anechoic chamber using a surrounding spherical microphone array. Section IV represents the acoustic source centering, comparing a previous acoustic centering algorithm ${ }^{18}$ to a recently developed one ${ }^{19}$ in order to increase the frequency range that allows for correct source centering. Section $\mathrm{V}$ describes an averaging procedure of the radiation patterns in third-octave bands. Section VI gives an overview of the instruments with their specific note range at which they were recorded. Section VII presents an analysis of the acoustic radiation excitation points from the instruments using the acoustic source centering algorithm. Section VIII discusses the effect of the human player on the radiation pattern by comparing radiation pattern recorded using different methods.

\section{CALIBRATION}

A spherical array of 32 Sennheiser KE4-211-2 (Am Labor 1, Wedemark, Germany) electret microphones covering a
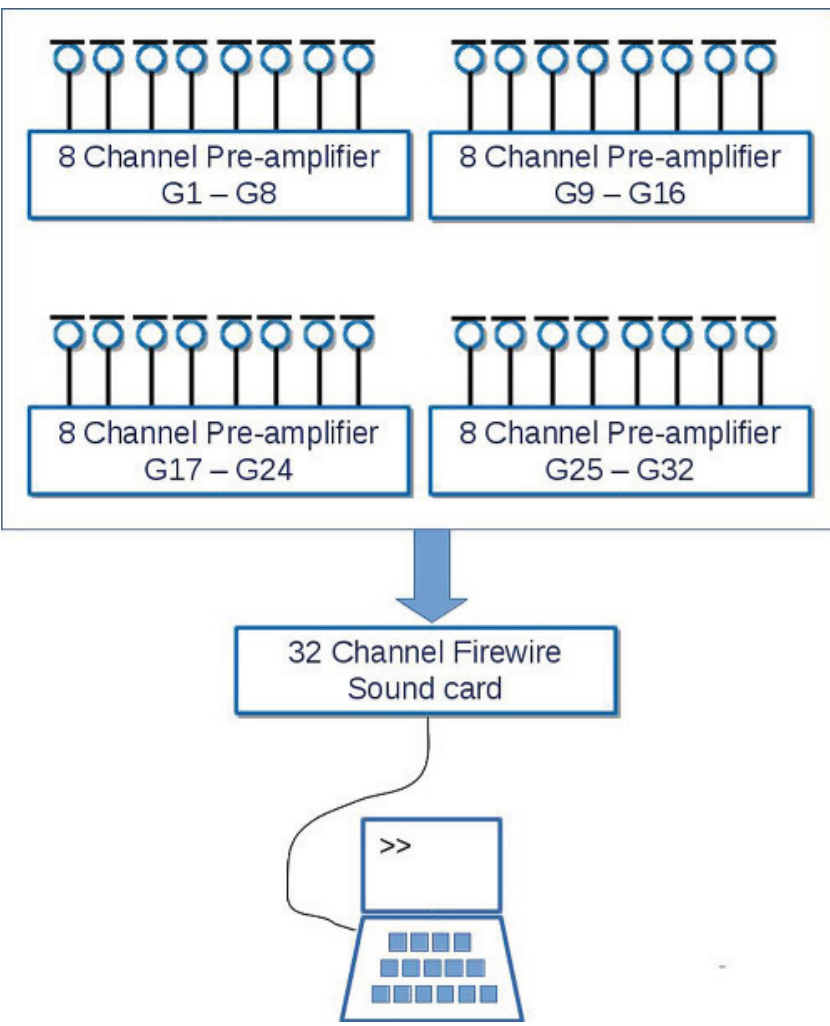

FIG. 2. (Color online) Electrical calibration results in different gain values $G_{1}-G_{32}$. frequency range from below $40 \mathrm{~Hz}$ to about $18 \mathrm{kHz}$ has been calibrated. Four 8-channel RME OctaMic (Am Pfarderling 60, Haimhausen, Germany) (microphone preamplifier/AD-converter) were used in order to connect 32 microphones to symmetrical input amplifiers and to apply AD-conversion with 24 bit resolution at a sampling frequency of $48 \mathrm{kHz}$. The four Alesis digital audio tape (ADAT) (Company Alesis, Cumberland, RI) outputs of the microphone pre-amplifiers were connected to a PC running Steinberg Media Technologies GmbH Nuendo (Frankenstr. 18 b, Hamburg, Germany) as a recording tool. The recording chain from the microphones to the computer is depicted in Fig. 2. This scheme results in 32 different gain values, appearing as $G_{1 \ldots 32}$ in Fig. 2.

A calibration process has been performed each time the gain factor of the measurement chain was changed. To measure all individual 32 gain factors, a sweep sine signal was fed to all the 32 input ports simultaneously, and the impulse response of the entire measurement chain was captured. The gain values were changed during the recording of the 41 instruments with respect to the dynamic level of the instrument sound, so to ensure that neither overload nor under-run of the inputs would occur. After the calibration of the electrical measurement chain (microphone input), a pistonphone calibrator [Brüel \& Kjær (B\&K) 4230 (Skodsborgvej 307, Nærum, Denmark), $94 \mathrm{~dB} @ 1 \mathrm{kHz}$ ] was used with the most accessible microphone in the sphere to obtain the absolute sensitivity of the measurement setup. This procedure required the knowledge of the individual microphone transfer functions, as described next.

The individual sensitivities of all microphones were measured in a substitution measurement procedure as depicted in Fig. 3. A loudspeaker with good coverage of the frequency range from $50 \mathrm{~Hz}$ to $20 \mathrm{kHz}$ was used to excite a

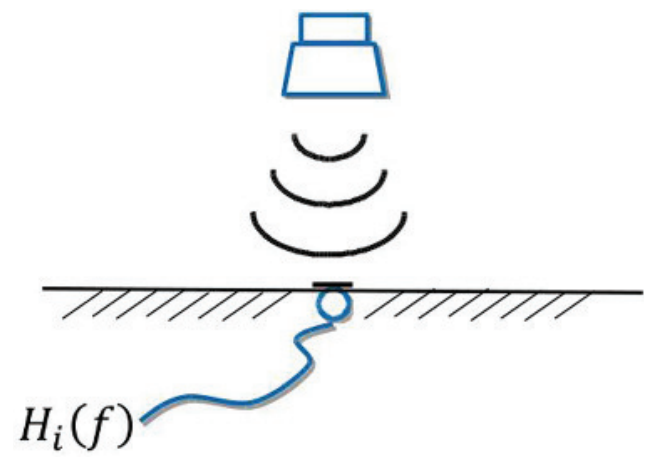

FIG. 3. (Color online) Transfer function measurement of an individual microphone. 


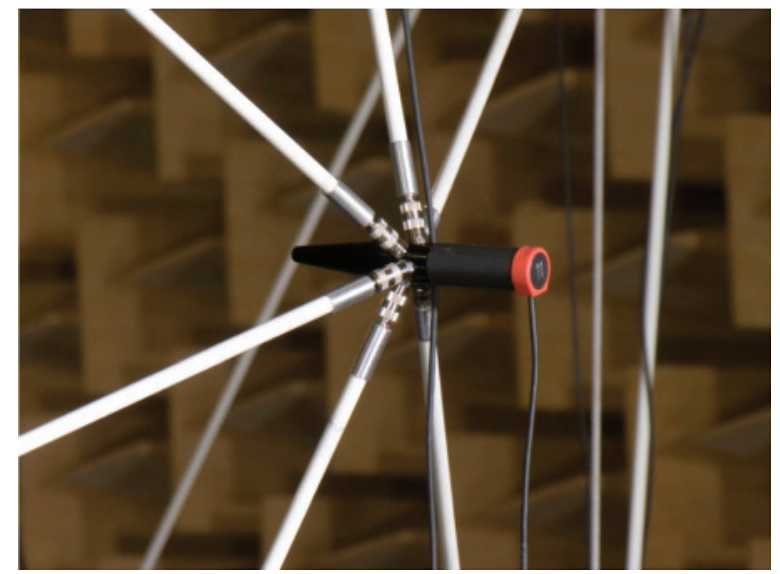

FIG. 4. (Color online) Supporting beam-structure (six beams) for one of the microphones.

sine sweep signal and a reference microphone (B\&K $1 / 4$ in. type 4939) was used to measure the sound pressure created at a distance of $1 \mathrm{~m}$. Then, all 32 microphones of the sphere where subsequently placed at the position of the reference microphone, and the measurement was repeated with the same signal. The result is a set of the microphone transfer functions, $\left\{H_{k}(\cdot)\right\}_{k=1}^{32}$, derived by a complex spectral division of the microphone measurement with the reference measurement. These transfer functions, showing the frequency dependent deviation from the reference microphone, were used to normalize the recordings of each individual microphone.

This calibration method assumes that the recording space is ideally anechoic. However, if there is the slightest amount of reflected energy from the equipment or supporting structures, the resulting responses at the spatially positioned microphones will vary from each other. To include such possible effects in the calibration, the microphone responses should usually be measured in their actual positions, as shown in Fig. 4. However, a free field calibration of the microphones has been performed showing a flat frequency response within a frequency range up to $2 \mathrm{kHz}$. Above this frequency, diffraction artifacts from the supporting structure became significant.

For a better understanding of this phenomenon, a boundary element method (BEM) simulation of the structure has been performed showing a more detailed analysis of the angle dependent diffraction behavior, as can be seen in the BEM simulation response in Fig. 5. Hence, the frequency response up to $3 \mathrm{kHz}$ is not affected by this problem, and therefore the analysis of the radiation pattern of the musical instruments is performed below this frequency limit.

To conclude, in order to compensate for all the possible errors, the following three steps have been taken seriously:

(1) Calibration of the frequency response for each individual microphone performed with substitution method as described.

(2) Evaluation of the diffraction influence of the setup.

(3) "In situ" calibration of the overall channel sensitivity (microphone to AD-converter output) using a $94 \mathrm{~dB} 1 \mathrm{kHz}$ pistonphone $(\mathrm{B} \& \mathrm{~K})$.

In the final result, the individual frequency response and the in situ calibration were taken into account, whereas the diffraction was neglected due to the limited influence in the frequency range of interest.

\section{RECORDING}

The directional sound from each musical instrument was recorded using a light-weight transportable surrounding spherical microphone array in the large fully anechoic chamber of TU Berlin $\left(V=1070 \mathrm{~m}^{3}, f_{g}=63 \mathrm{~Hz}\right)$. The entire setup can be seen in Fig. 6. The surrounding spherical array was of $2.1 \mathrm{~m}$ radius, and consisted of 32 microphones located on the faces of a truncated icosahedron. The microphones were held by 90 lightweight but nevertheless robust fiber-glass sticks. An adjustable chair has been used in order to place the musician approximately at the geometrical center. The musicians were facing toward the positive $x$ axis direction and the head of each musician was located near the physical center of the microphone array.

Individual notes were separately recorded. For the identification of fundamental and overtone frequencies, only the stationary parts of the recorded notes are of interest. Therefore, these were manually extracted, multiplied by a Hamming window, and a fast Fourier transform was calculated for each windowed steady signal, from which peaks were detected using the findpeaks MATLAB function. Figure 7 shows an example of the peak detection for the signal recorded from an authentic trumpet, playing the note A3 (according to ISO 16:1975 notation $^{22}$ ) in ff, using four different microphones.

For each musical instrument, the first ten peaks were detected and associated with the first ten partials (a fundamental frequency and nine following overtones). The complex amplitude of the partials were saved in three-dimensional (3D) tensors

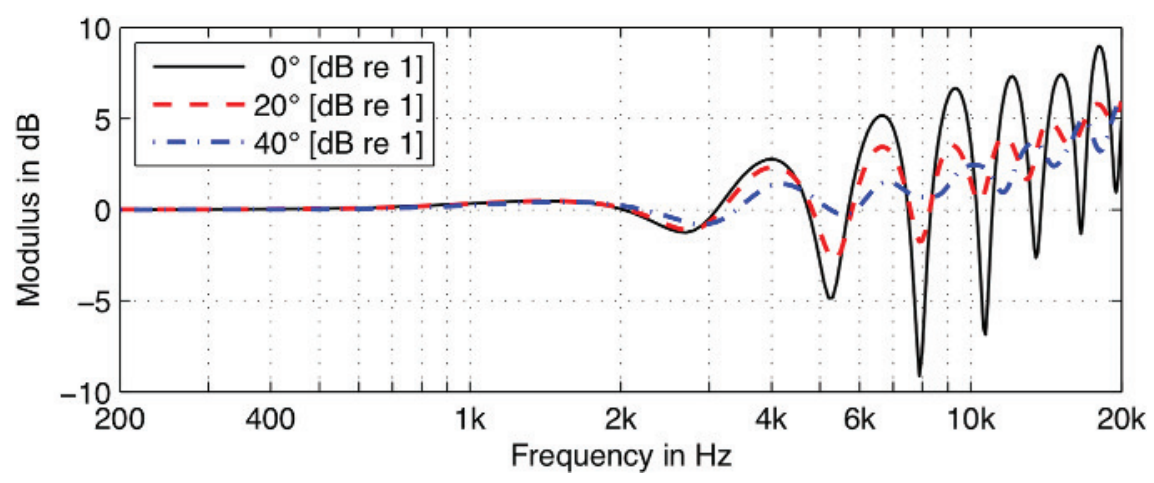

FIG. 5. (Color online) Microphone structure response from BEM simulation. 


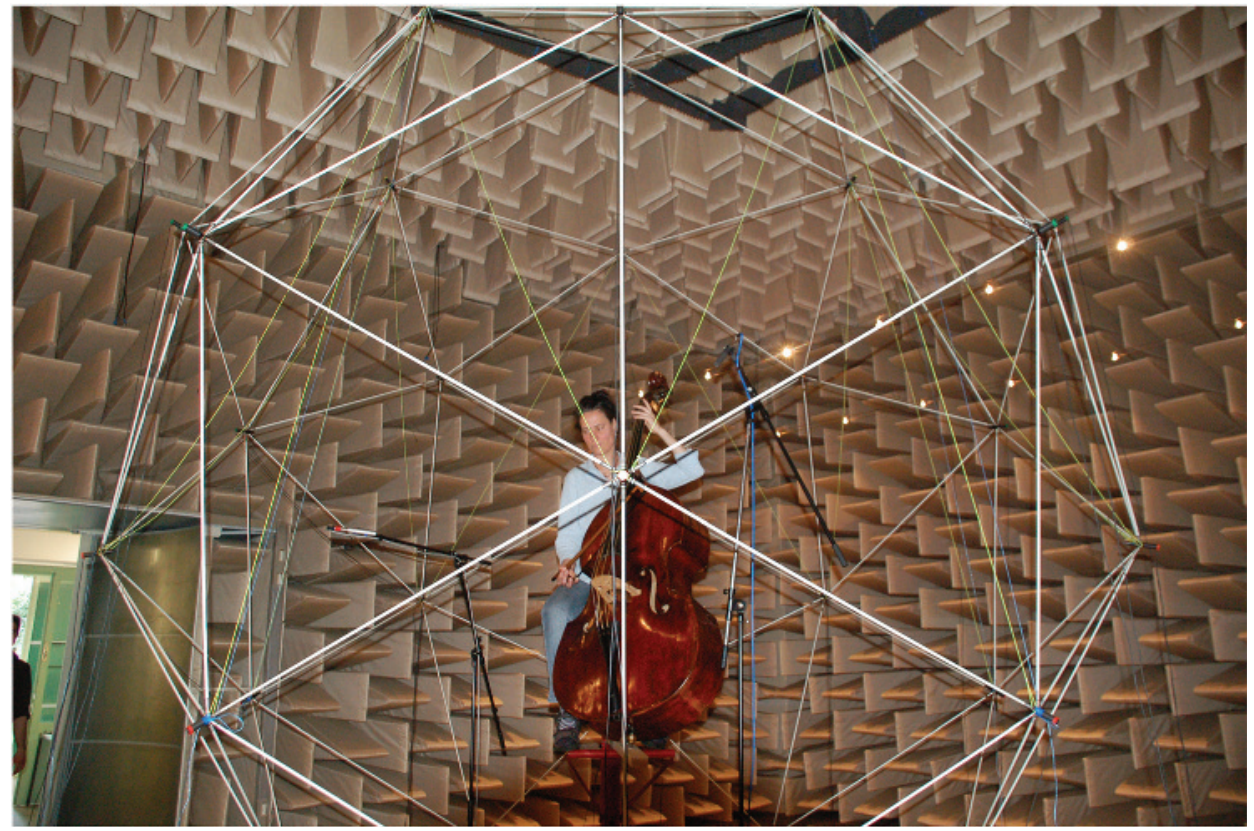

FIG. 6. (Color online) Spherical microphone array surrounding the musicians.

$$
\tilde{\tilde{\mathbf{A}}} \in \mathbb{C}^{N_{\text {note }}} \times \mathbb{C}^{N_{\mathrm{pa}}} \times \mathbb{C}^{N_{\text {ch }}},
$$

where $N_{\text {note }}, N_{\text {pa }}$, and $N_{\text {ch }}$ are the numbers of the played notes, partials (10), and channels (32), respectively. Also for each musical instrument, the tensor

$$
\mathbf{F} \in \mathbb{R}^{N_{\text {note }}} \times \mathbb{R}^{N_{\text {pa }}} \times \mathbb{R}^{N_{\text {ch }}},
$$

contains the actual frequencies where the peaks were detected.

The calibration stage described in Sec. II resulted in gain differences between the signals at all the 32 channels, as well as different frequency responses. Although usually this compensation would have been performed as a first step of the frequency analysis, in this case it was found more practical to only compensate the signals at their detected peaks in the frequency domain, because of the clear harmonic pattern of the sound that is produced using musical instruments.

The compensation of the electrical gain differences was performed using

$$
\tilde{A}_{i j k}=G_{k}^{-1} \tilde{\tilde{A}}_{i j k}
$$

where $k=1 \ldots N_{\mathrm{ch}}$ is the channel index, with $G_{k}$ its corresponding electrical gain as described in Sec. II, and $i=1 \ldots N_{\text {note }}$ and $j=1 \ldots N_{\mathrm{pa}}$ is the played note index and the partial index, respectively. The acoustical compensation was then performed using

$$
A_{i j k}=H_{k}^{-1}\left(F_{i j k}\right) \tilde{A}_{i j k},
$$

where $H_{k}$ is the frequency response of the $k$ th microphone, as described in Sec. II.

\section{ACOUSTIC SOURCE CENTERING}

Acoustic source centering is the most mathematically demanding stage in the generation of the radiation pattern database, here presented in brevity, but extensively elaborated in the works of Hagai et al. ${ }^{18}$ and of Shabtai and Vorl änder. ${ }^{19}$
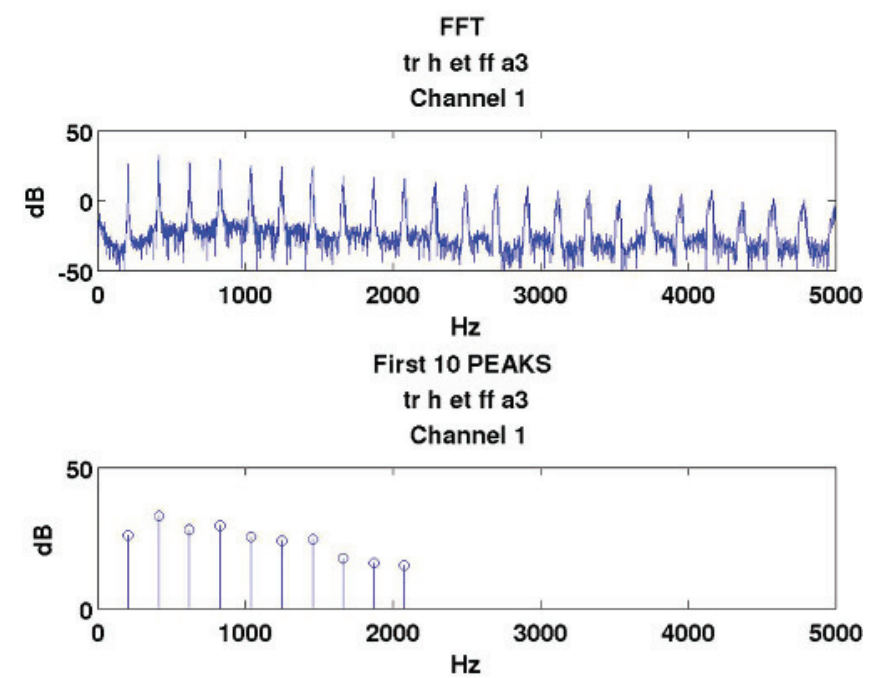

(a)

FFT

tr h et ff a3

Channel 32

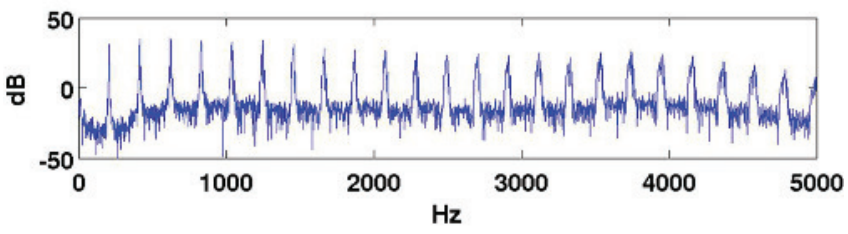

First 10 PEAKS

tr $\mathrm{h}$ et $\mathrm{ff}$ a3

Channel 32

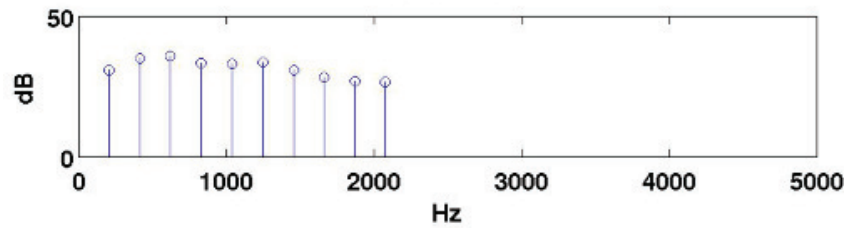

(b)

FIG. 7. (Color online) Detection of fundamental frequency and overtones in channels (a) 1 and (b) 32 for an authentic trumpet playing the note A3 at fortissimo dynamic level. 
Acoustic source centering is performed in order to compensate for the misalignment of the origin of the coordinate system (or the physical center of the spherical microphone array) with the acoustic center of the musical instrument. Although the acoustic center is not unique, a mathematical representation of a single acoustic center is required in order to model the radiation pattern. Furthermore, it is preferred to select an acoustic center that reduces the order of the radiation pattern in the spherical harmonics domain in order to improve the efficiency of its implementation. ${ }^{18}$

The radiation pattern is modeled as the pressure function in a spherical coordinate system. The pressure function at a wave number $k$ is defined on a surface of a sphere of radius $r$ with an elevation angle $\theta$ and an azimuth angle $\phi$, using the order-limited spherical Fourier series in the form of

$$
p(k, r, \theta, \phi)=\sum_{n=0}^{N} \sum_{m=-n}^{n} c_{n m}(k) h_{n}(k r) Y_{n}^{m}(\theta, \phi),
$$

where $c_{n m}(k)$ are coefficients that represent the particle velocity at the surface of the source at wave number $k$, and $h_{n}(k r)$ is the $n$th order spherical Hankel function of the first kind. Every base function $Y_{n}^{m}(\cdot, \cdot)$ is referred to as the spherical harmonic of order $n$ and degree $m$, given by

$$
Y_{n}^{m}(\theta, \phi) \triangleq \sqrt{\frac{2 n+1}{4 \pi} \frac{(n-m) !}{(n+m) !}} P_{n}^{m}(\cos \theta) e^{i m \phi},
$$

where $P_{n}^{m}(\cdot)$ is the associated Legendre function ${ }^{23}$ of order $n$ and degree $m$.

A vector of pressure values recorded by an array of $Q$ microphones at locations $\left\{\mathbf{r}_{q}=\left(r_{q}, \theta_{q}, \phi_{q}\right)\right\}_{q=1}^{Q}$ is defined by

$$
\mathbf{p}(k) \triangleq\left[p\left(k, \mathbf{r}_{1}\right), p\left(k, \mathbf{r}_{2}\right), \ldots, p\left(k, \mathbf{r}_{Q}\right)\right]^{T}
$$

and a steering matrix of size $Q \times(N+1)^{2}$ by

$$
\mathbf{H}(k) \triangleq\left[\begin{array}{lllll}
Y_{0}^{0}\left(\theta_{1}, \phi_{1}\right) h_{0}\left(k, r_{1}\right) & Y_{1}^{-1}\left(\theta_{1}, \phi_{1}\right) h_{1}\left(k, r_{1}\right) & Y_{1}^{0}\left(\theta_{1}, \phi_{1}\right) h_{1}\left(k, r_{1}\right) & \ldots & Y_{N}^{N}\left(\theta_{1}, \phi_{1}\right) h_{N}\left(k, r_{1}\right) \\
Y_{0}^{0}\left(\theta_{2}, \phi_{2}\right) h_{0}\left(k, r_{2}\right) & Y_{1}^{-1}\left(\theta_{2}, \phi_{2}\right) h_{1}\left(k, r_{2}\right) & Y_{1}^{0}\left(\theta_{2}, \phi_{2}\right) h_{1}\left(k, r_{2}\right) & \ldots & Y_{N}^{N}\left(\theta_{2}, \phi_{2}\right) h_{N}\left(k, r_{2}\right) \\
\vdots & \vdots & \vdots & \ddots & \vdots \\
Y_{0}^{0}\left(\theta_{Q}, \phi_{Q}\right) h_{0}\left(k, r_{Q}\right) & Y_{1}^{-1}\left(\theta_{Q}, \phi_{Q}\right) h_{1}\left(k, r_{Q}\right) & Y_{1}^{0}\left(\theta_{Q}, \phi_{Q}\right) h_{1}\left(k, r_{Q}\right) & \ldots & Y_{N}^{N}\left(\theta_{Q}, \phi_{Q}\right) h_{N}\left(k, r_{Q}\right)
\end{array}\right]
$$

The particle-velocity coefficients vector

$$
\mathbf{c}_{\mathrm{SH}}(k) \triangleq\left[c_{00}(k), c_{1-1}(k), \ldots, c_{N N}(k)\right]^{T},
$$

can be estimated in the least-squares sense using

$$
\mathbf{c}_{\mathrm{SH}}(k)=\mathbf{H}^{\dagger}(k) \mathbf{p}(k),
$$

where the dagger sign $(\dagger)$ denotes the pseudo-inverse operator defined by $\mathbf{H}^{\dagger}=\left(\mathbf{H}^{H} \mathbf{H}\right)^{-1} \mathbf{H}^{H}$.

Matrix $\mathbf{H}(k)$ can be recalculated for an origin point that is translated by $\Delta \mathbf{r}$, with the new location vectors $\mathbf{r}_{q}^{\prime}(\Delta \mathbf{r})$, resulting from this translation. Using the original, not translated, pressure vector $\mathbf{p}(k)$, coefficients $c_{n m}(k)$ can be recalculated for the translation using

$$
\mathbf{c}_{\mathrm{SH}}(k \mid \Delta \mathbf{r})=\mathbf{H}^{\dagger}(k \mid \Delta \mathbf{r}) \mathbf{p}(k) .
$$

For each wave number $k$, the acoustic center $\mathbf{r}_{c}(k)$ is calculated using an objective function $J(\cdot \mid k)$, so that

$$
\mathbf{r}_{c}(k)=\arg \min _{\Delta \mathbf{r}} J(\Delta \mathbf{r} \mid k) .
$$

Hagai et al. ${ }^{18}$ suggested to use a center-of-mass concept based objective function in the form of

$$
J_{\mathrm{c}}(\Delta \mathbf{r} \mid k)=\frac{\sum_{n=0}^{N} \sum_{m=-n}^{n} n\left|c_{n m}(k \mid \Delta \mathbf{r})\right|^{2}}{\sum_{n=0}^{N} \sum_{m=-n}^{n}\left|c_{n m}(k \mid \Delta \mathbf{r})\right|^{2}}
$$

A later development by Shabtai and Vorländer ${ }^{19}$ showed that a phase symmetrical propagation based objective function in the form of

$$
J_{\mathrm{p}}(\Delta \mathbf{r} \mid k)=-\frac{\sum_{n=0}^{N}\left|c_{n 0}(k, \Delta \mathbf{r})\right|^{2}}{\sum_{n=0}^{N} \sum_{m=-n}^{n}\left|c_{n m}(k, \Delta \mathbf{r})\right|^{2}},
$$

converges better for natural sources, and in a wider range of frequencies. Furthermore, $J_{\mathrm{p}}(\Delta \mathbf{r} \mid k)$ can be calculated on separate planes to speed up calculation time, whereas $J_{\mathrm{c}}(\Delta \mathbf{r} \mid k)$ must be calculated on the entire 3D space. The objective function $J_{\mathrm{p}}(\Delta \mathbf{r} \mid k)$ is designed for source centering in the $x-y$ plane. In order to complete the centering, another scanning is performed in the $y-z$ domain, which requires a rotation procedure that is given in the work of Shabtai and Vorländer. ${ }^{19}$

For a comparison of the acoustic source centering results in different parallel planes when $J_{c}$ and $J_{p}$ are used for a trumpet and a guitar, the reader is referred to the recent work published by Shabtai and Vorländer. ${ }^{19}$ In this work, the performance of the acoustic source centering is compared for musical instruments from different categories in Sec. VI. For the generation of the acoustic radiation database that is described in this work, acoustic centering has been applied using $J_{c}$ up to the frequency of $0.5 \mathrm{kHz}$, and using $J_{p}$ 
for frequencies between 0.5 and $1 \mathrm{kHz}$. Acoustic source centering has not been applied to the radiation pattern at frequencies higher than $1 \mathrm{kHz}$ because of spatial aliasing, as described in the previous acoustic centering works. ${ }^{18,19}$

\section{AVERAGING IN THIRD-OCTAVE BANDS}

In order to derive a compact representation of radiation patterns that can easily be implemented in room-acoustics simulations, a single radiation pattern has been created for each frequency band. The radiation pattern in a certain frequency band depends on the played note, as can be noticed for example, in the case of woodwind instruments, where the sound is radiated through the open finger holes. However, overtone frequencies that are related to different note fundamental frequencies may fall into the same frequency band. Therefore, the coefficients $p_{n m}(f)$ were averaged over all frequencies $f$ inside the frequency band.

Hence, defining the $i$ th band set of partial frequencies

$$
\begin{gathered}
A_{i}=\left\{f \in \operatorname{band}(i) \mid \exists \text { fundamental frequency } f_{0},\right. \\
\left.\quad l \in \mathbb{N} \mid f=l f_{0}\right\},
\end{gathered}
$$

the averaged radiation coefficients are calculated using

$$
p_{n m}^{i \text { th band }}=\frac{1}{\left|A_{i}\right|} \sum_{f \in A_{i}} p_{n m}(f) .
$$

Thus, the partials $l f_{0}$ of all potential note fundamental frequencies $f_{0}$ are invoked. The radiation pattern can be extracted to the spatial domain from the $p_{n m}^{i \text { th band }}$ values using

$$
p_{i \text { th band }}(\theta, \phi)=\sum_{n=0}^{N} \sum_{m=-n}^{n} p_{n m}^{i \text { th band }} Y_{n}^{m}(\theta, \phi) \text {, }
$$

with any desired resolution for $\theta$ and $\phi$. The extracted radiation pattern can then be used in a computer simulation program that calculates directional RIRs, e.g., ODEON or RAVEN. The last is facilitated by using the open directional audio file format package. ${ }^{24}$

\section{THE INSTRUMENT DATABASE}

The acoustic radiation pattern of 41 modern and authentic instruments of the symphonic orchestra has been measured in the above mentioned method. For each instrument, several full chromatic scales have been recorded at different dynamic levels of playing. Professional players have been instructed to play pianissimo ("as soft as possible, without the sound tearing") and fortissimo ("as loud as possible, without the tone getting unpleasant"), to avoid any frequency modulations caused by vibrato, and to avoid amplitude modulations caused by other expressive techniques. The dynamic style has not been double checked. The notes were played non-legato in order to bind a steady signal with two silent parts beforehand and afterwards, facilitating the independent analysis of the directional response at each tone. There was no strict requirement to the length of the notes, only that they are being played for few seconds. Register change was permitted and the used register was not tracked during the recording process.

Table I presents a list of the instruments as well as the note ranges, which might be slightly different for the different dynamic levels. From the acoustic radiation point of view, a preliminary sorting of the instruments can be made into three categories:

- Category 1: Instruments with one expected radiation point. Mainly includes the brass instruments, where only sound from the bell opening is considered for radiation.

- Category 2: Instruments with several expected radiation points. Mainly includes woodwind instruments, with sound radiated by the bell, the fingering holes, and the

\begin{tabular}{|c|c|c|c|c|c|c|}
\hline \multirow{2}{*}{$\begin{array}{l}\text { Instrument } \\
\text { Bassoon }\end{array}$} & \multirow{2}{*}{$\frac{\text { Era }}{m}$} & \multicolumn{2}{|c|}{ Scale@ff } & \multicolumn{2}{|c|}{ Scale@pp } & \multirow{2}{*}{$\frac{\text { Category }}{2}$} \\
\hline & & A\#1 & E5 & A\#1 & E5 & \\
\hline Contrabassoon & $\mathrm{m}$ & A\#0 & D\#3 & A\#0 & F3 & 2 \\
\hline Natural trumpet & $\mathrm{a}$ & D3 & D6 & D3 & D6 & 1 \\
\hline Trumpet & $\mathrm{m}$ & $\mathrm{F} \# 3$ & F6 & F\#3 & F6 & 1 \\
\hline Tenor trombone & $\mathrm{m}$ & G1 & F5 & G1 & F5 & 1 \\
\hline Bass trombone & $\mathrm{m}$ & E1 & F4 & E1 & F4 & 1 \\
\hline Viola & $\mathrm{m}$ & $\mathrm{C} 3$ & F7 & $\mathrm{C} 3$ & F7 & 3 \\
\hline Tuba & $\mathrm{m}$ & G\#0 & $\mathrm{C} 5$ & F0 & E5 & 1 \\
\hline Transverse flute & $\mathrm{m}$ & B3 & A\#6 & B3 & D7 & 2 \\
\hline Cello & $\mathrm{m}$ & $\mathrm{C} 2$ & G6 & $\mathrm{C} 2$ & G6 & 3 \\
\hline Oboe & $\mathrm{m}$ & A\#3 & A6 & A\#3 & $\mathrm{F} \# 6$ & 2 \\
\hline English horn & $\mathrm{m}$ & E3 & $\mathrm{G} \# 5$ & E3 & G\#5 & 1 \\
\hline Double action harp & $\mathrm{m}$ & $\mathrm{C} 0$ & F\#6 & $\mathrm{CO}$ & F\#6 & 3 \\
\hline Double bass & $\mathrm{m}$ & E1 & E5 & E1 & D\#5 & 3 \\
\hline French horn & $\mathrm{m}$ & D2 & F\#5 & D2 & G5 & 1 \\
\hline Clarinet & $\mathrm{m}$ & D3 & A\#6 & D3 & A\#6 & 2 \\
\hline Bass clarinet & $\mathrm{m}$ & A\#1 & $\mathrm{D \# 5}$ & A\#1 & D\#5 & 2 \\
\hline Violin & $\mathrm{a}$ & G3 & C7 & G3 & C7 & 3 \\
\hline Classic oboe & $\mathrm{a}$ & $\mathrm{C} 4$ & D\#6 & $\mathrm{C} 4$ & D\#6 & 2 \\
\hline Romantic oboe & $\mathrm{a}$ & B3 & C6 & D4 & G5 & 2 \\
\hline Soprano & - & G3 & G\#5 & G3 & G\#5 & 1 \\
\hline Classic bassoon & $\mathrm{a}$ & A\#1 & C5 & A\#1 & C5 & 2 \\
\hline Baroque bassoon & $\mathrm{a}$ & A1 & F\#4 & A1 & $\mathrm{F} \# 4$ & 2 \\
\hline Dulcian & $\mathrm{a}$ & $\mathrm{C} 2$ & G4 & $\mathrm{C} 2$ & G4 & 2 \\
\hline Timpani & $\mathrm{m}$ & $\mathrm{C} 0$ & $\mathrm{C} 0$ & $\mathrm{C} 0$ & $\mathrm{CO}$ & 3 \\
\hline Pedal timpani & $\mathrm{m}$ & $\mathrm{C} 0$ & $\mathrm{C} 0$ & $\mathrm{C} 0$ & $\mathrm{CO}$ & 3 \\
\hline Natural horn & $\mathrm{a}$ & A1 & B4 & A1 & B4 & 1 \\
\hline Cello & $\mathrm{a}$ & $\mathrm{C} 2$ & D5 & $\mathrm{C} 2$ & D5 & 3 \\
\hline Acoustic guitar & $\mathrm{m}$ & $\mathrm{C} 2$ & B5 & E2 & B5 & 3 \\
\hline Viola & $\mathrm{a}$ & $\mathrm{C} 3$ & C6 & $\mathrm{C} 3$ & C6 & 3 \\
\hline Keyed flute & $\mathrm{a}$ & $\mathrm{C \# 4}$ & A6 & $\mathrm{C \# 4}$ & A6 & 2 \\
\hline Baroque transverse flute & $\mathrm{a}$ & D4 & F\#6 & D4 & A\#6 & 2 \\
\hline Clarinet & $\mathrm{a}$ & D3 & F6 & D3 & F6 & 2 \\
\hline Basset horn & $\mathrm{a}$ & $\mathrm{F} 2$ & A\#5 & $\mathrm{F} 2$ & A\#5 & 1 \\
\hline Alto trombone & $\mathrm{a}$ & D2 & $\mathrm{D} \# 5$ & $\mathrm{C} \# 2$ & D\#5 & 1 \\
\hline Violin & $\mathrm{m}$ & G3 & G6 & G3 & G6 & 3 \\
\hline Double bass & a & E1 & E3 & E1 & E3 & 3 \\
\hline Bass trombone & $\mathrm{a}$ & B1 & B4 & $\mathrm{C} 1$ & B4 & 1 \\
\hline Tenor trombone & $\mathrm{a}$ & E1 & D5 & E1 & D5 & 1 \\
\hline Alto saxophone & $\mathrm{m}$ & $\mathrm{C} \# 2$ & $\mathrm{C} \# 5$ & $\mathrm{C} \# 2$ & $\mathrm{C \# 5}$ & 2 \\
\hline Tenor saxophone & $\mathrm{m}$ & B1 & A4 & B1 & A4 & 2 \\
\hline
\end{tabular}
mouth piece. In addition, different fingering options (and

TABLE I. List of modern (m) and authentic (a) era instruments for which radiation pattern was measured. 
thus, radiation patterns) may be possible for the same tone.

- Category 3: Instruments with a full body radiating sound. String instruments belong to this group. In this group, the acoustic radiation depends on the modal vibration of the body and may be very complicated. The radiation pattern, therefore, might as well be very individual and a generalization is only allowed at the lower end of modal frequencies, if at all.

The category index to each instrument in Table I appears in the last column.

\section{ANALYSIS OF THE ACOUSTIC RADIATION EXCITATION POINTS}

In this section, an analysis of the acoustic radiation excitation points is performed for musical instruments from the different categories which were represented in Sec. VI, using the acoustic source centering algorithms defined in Sec. IV. First, a graphical analysis of the acoustic source centering objective function behavior is presented in Sec. VII A for a bass trombone and tuba as representative instruments from category 1 , bassoon and clarinet from category 2 , and viola and cello from category 3 . The graphical analysis of the objective function behavior is performed at specific harmonic partial frequencies. Second, a statistical analysis of the local minimum points of the acoustic source centering objective function is performed at different low frequency bands, for which acoustic centering is applied to all instruments in the database.

\section{A. Graphical analysis}

Figures $8-10$ show the results of the acoustic source centering objective functions for the note F\#3 (fundamental frequency of $\approx 185 \mathrm{~Hz}$ ), at the third harmonic partial $(\approx 560 \mathrm{~Hz})$, for categories $1-3$, respectively. The objective functions $J_{c}$ and $J_{p}$ from Sec. IV are presented in the first and second row of the figures, respectively. Generally, the domain of these objective functions is the entire $3 \mathrm{D}$ volume, however only partially represented in the figures in three orthogonal planar cross sections. The left column shows the objective function results in the $x-y$ domain at $z=0$, the middle in $y-z$ domain at $x=0$, and the right in $x-z$ at $y=0$ is redundant but shown nevertheless for completion. In the bottom of each figure, the configuration of the instrument in the anechoic chamber at TU Berlin is shown, where the head of the player is roughly located at the physical center of the
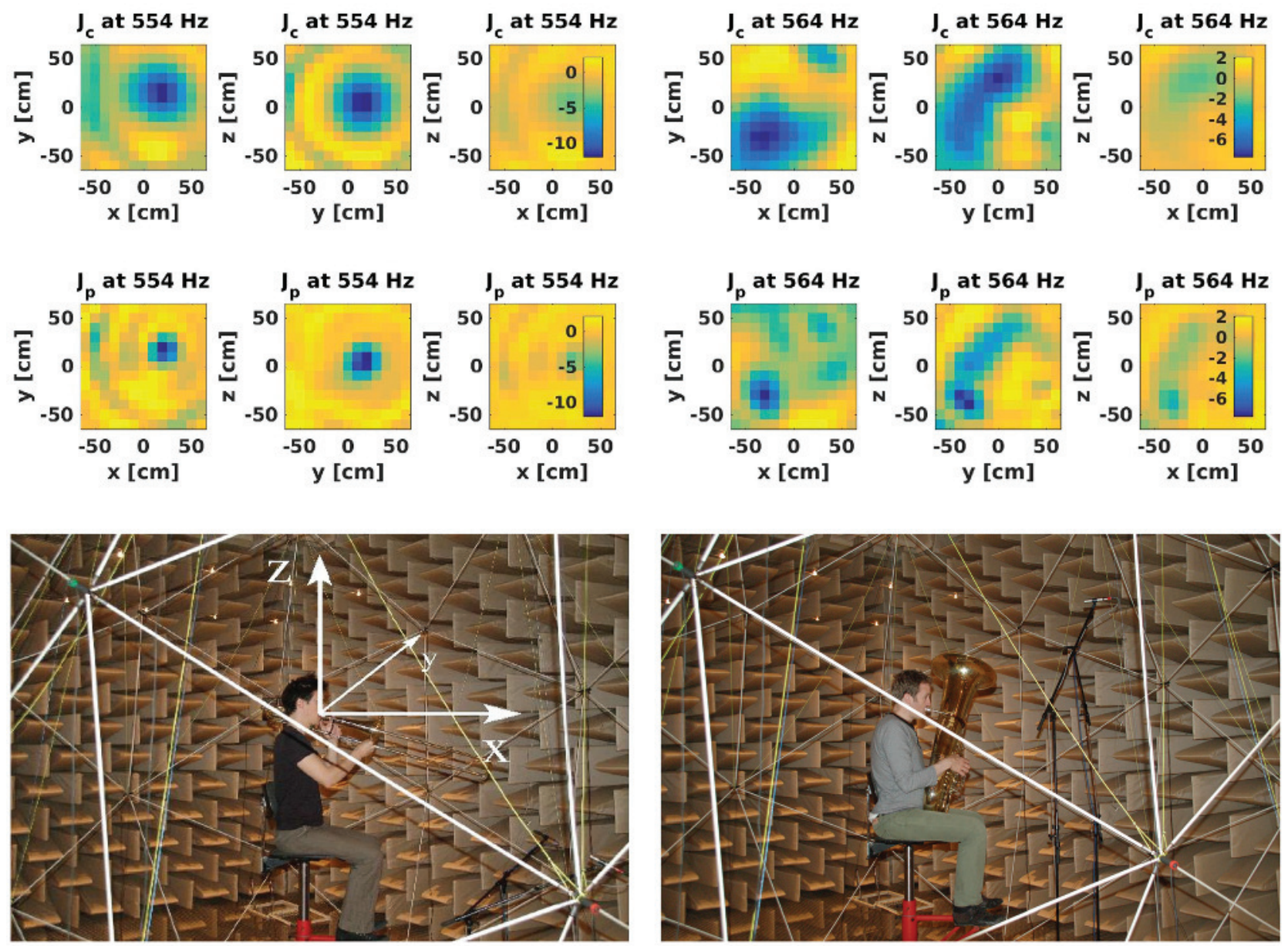

(a)

(b)

FIG. 8. (Color online) Acoustic centering algorithm for bass trombone (a) and tuba (b) from category 1. Color bar shows objective function values, normalized to use the same scale. 
surrounding spherical array of 32 microphones. The coordinate system is as follows: the player looks at the positive $x$ direction, the positive $y$ direction is stretched from the right ear of the player inside the page, and the positive $z$ direction is upwards, as can be seen in the bottom of Fig. 8(a).

For the acoustics centering results of musical instruments from category 1, shown in Fig. 8, the clear difference between the size of the sources can be viewed. While the objective functions in the case of the bass trombone [Fig. 8(a)] show a narrow descent toward a single clear acoustic center, in the case of the tuba [Fig. 8(b)] the descent of the objective functions smears according to the shape of the tuba itself, nevertheless showing a single acoustic center. However, the location of the acoustic center is different with respect to the physical body of the tuba. When the objective function $J_{c}$ is used, the minimum value appears at the bell, but when $J_{p}$ is used, the minimum value appears at the fingering location.

In the case of musical instruments from category 2, shown in Fig. 9, different excitation patterns can be found. In the case of the bassoon [Fig. 9(a)], two excitation sources seem to be detected using both objective functions, one at the bell extending upwards, and one toward the boot of the instrument, where several finger holes are located. In the case of the clarinet [Fig. 9(b)], a single acoustic center can be detected, again using both objective functions, downwards toward the location of the bell, as can be seen from the $z$ component.

For the musical instruments from category 3, shown in Fig. 10 , it is more difficult to find a clear convergence of the objective functions toward isolated local minima, which indicates that many excitation sources contribute to the generation of the acoustic radiation pattern, and an acoustic center seems not to naturally exist. However, in the case of the viola [Fig. 10(a)], the objective function $J_{p}$ detects that it is located at the left to the head of the player, as can be seen from the middle column of Fig. 10(a). This location of the viola in the $y$ axis could not be detected by using $J_{c}$. In the case of the cello [Fig. 10(b)], $J_{p}$ detects a local minimum in the $z$ axis, as can be seen in the middle and right columns of Fig. 10(b), which would have been less clearly detected if $J_{c}$ had been used. Furthermore, a roughly oval shape circumference of the cello can be detected in the $y-z$ plane by the results of $J_{p}$ in the middle column of Fig. 10(b).

It might be observed, then, that for smaller brass instruments from category 1 (e.g., trumpet and trombones), the objective function converges to a single minimum point. This result is in line with the assumption that brass instruments should have single radiation points. However, if the brass instrument is very big (e.g., the tube), or if the bell is too far from the physical center of the microphone array, a minimum area is stretched in the shape of the instrument in
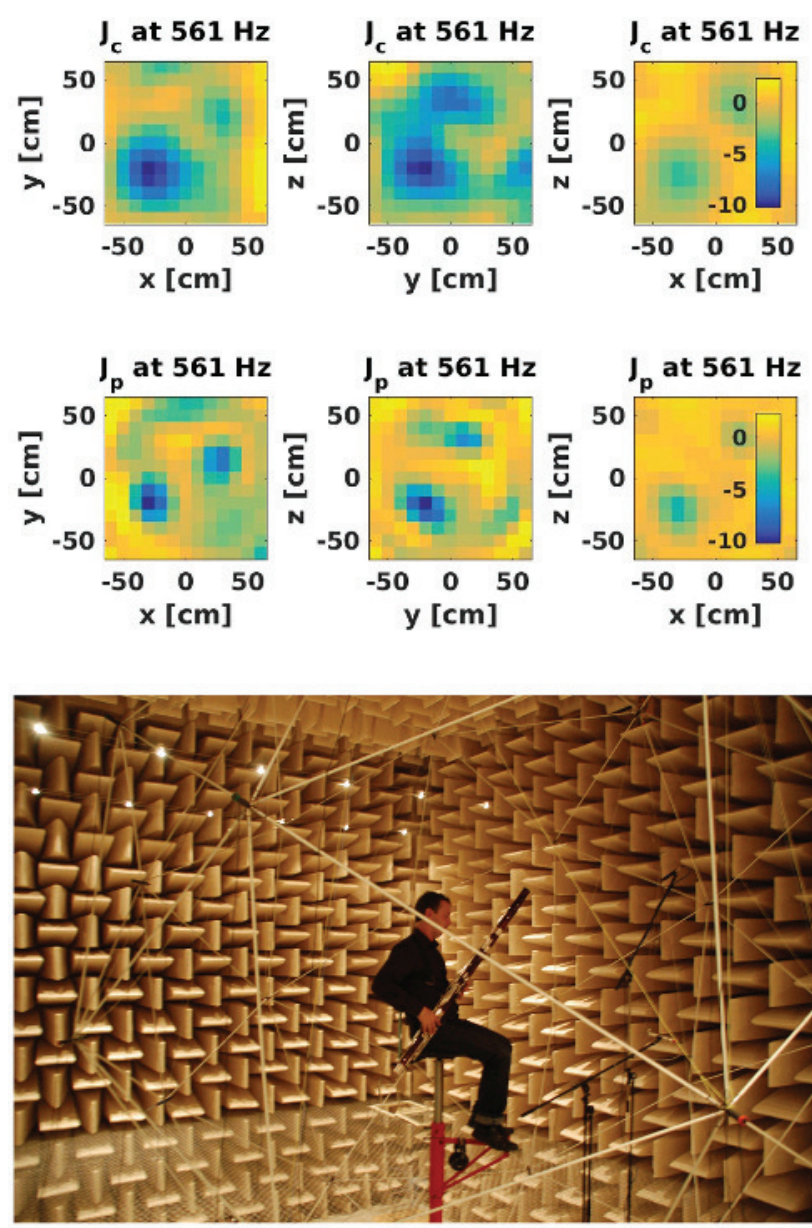

(a)
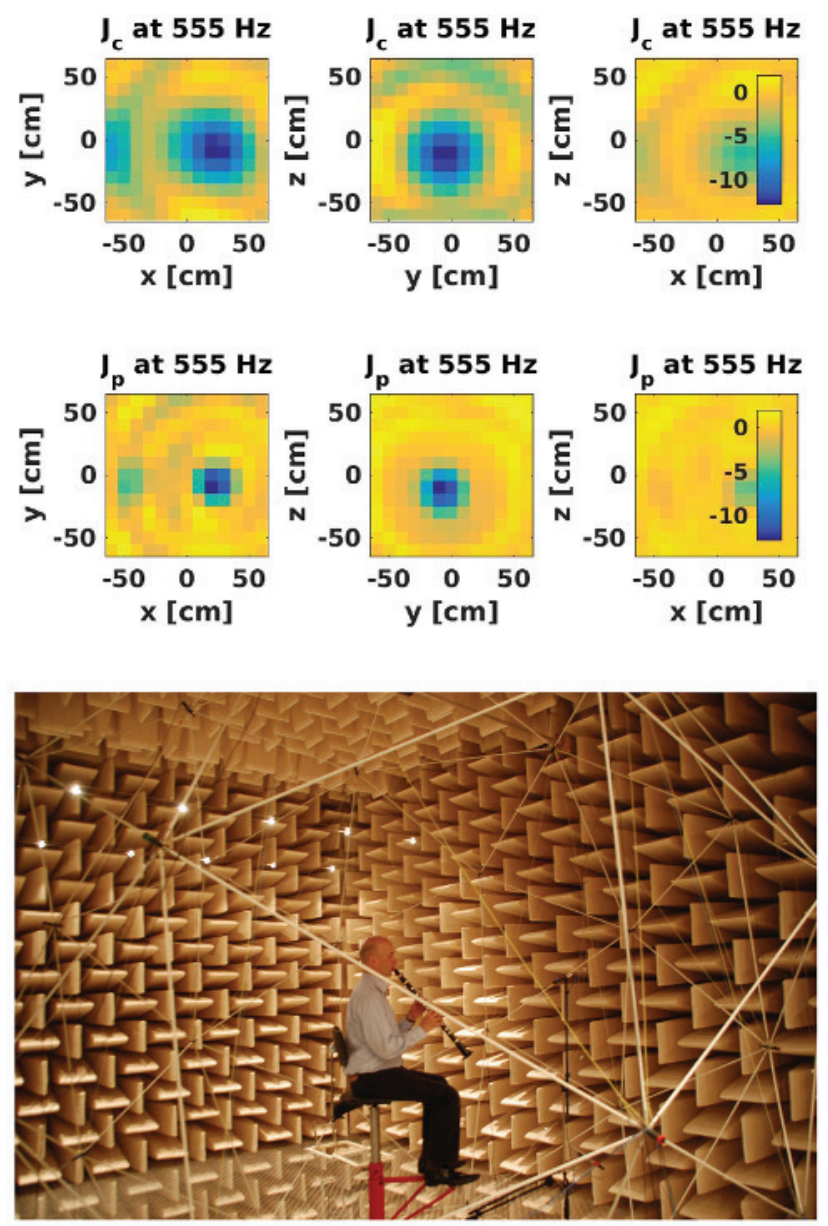

(b)

FIG. 9. (Color online) Acoustic centering algorithm for bassoon (a) and clarinet (b) from category 2. 

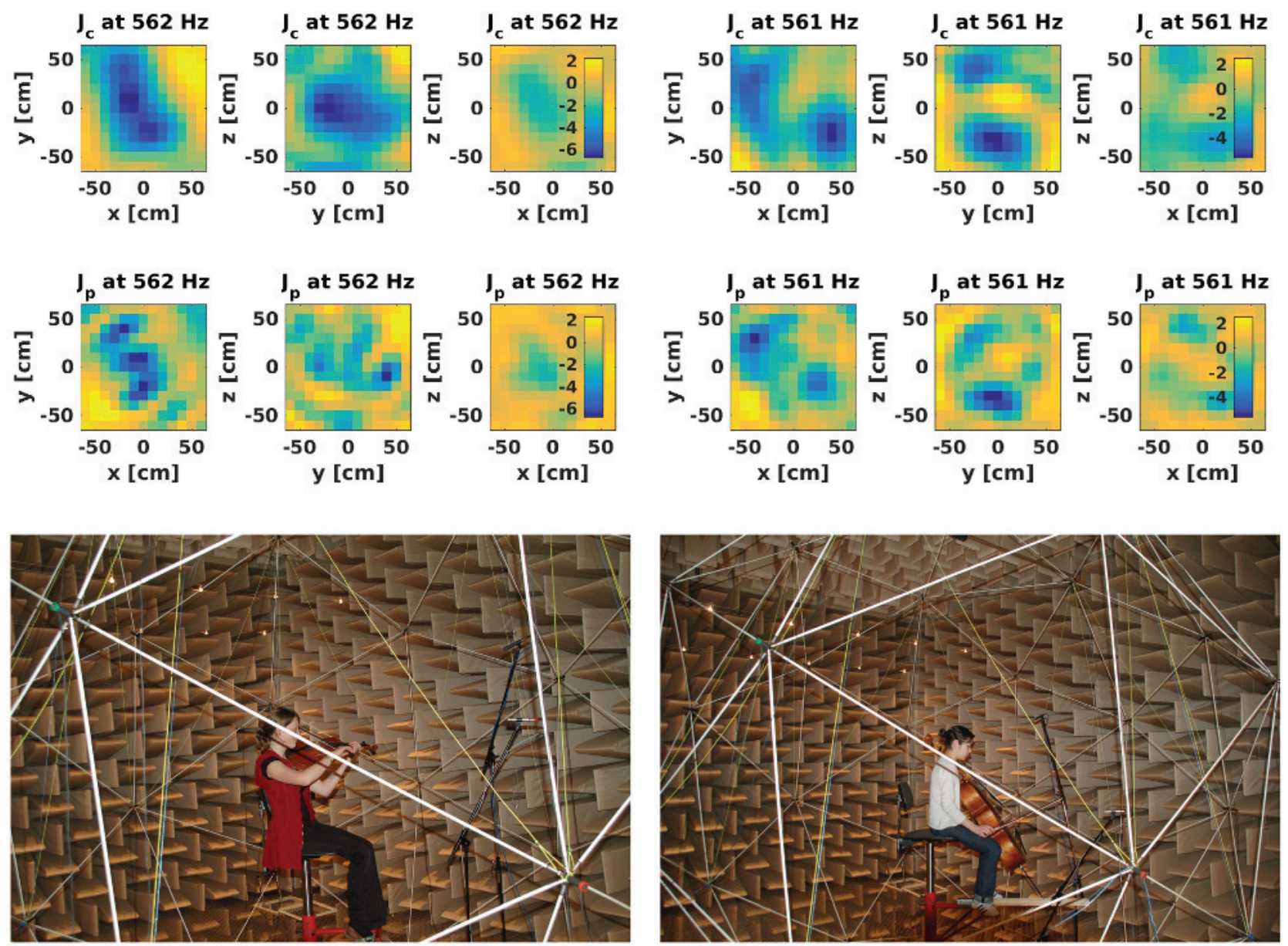

(a)

(b)

FIG. 10. (Color online) Acoustic centering algorithm for viola (a) and cello (b) from category 3.

addition to minimum around the bell location. This result is not surprising since if the bell is the source of radiation, then the centering objective function should show a sharper minimum at the bell location. For musical instruments of category 2, with several radiation points, the second source had only been found in the bassoon and not in the clarinet. For musical instruments with a full body radiating sound from category 3 , such as the string instruments, the acoustic source radiation objective function showed a complex map instead of clear minima, as expected; however, one of the objective function had shown clearer minima near the bridge of the instrument.

\section{B. Statistical analysis}

A statistical analysis of the number of local minimum points found in the acoustic source centering objective function is performed as a prediction to the number of expected excitation points. Although better predictors could be used, developing such a predictor is outside the scope of this work. For the analysis, data from all the instruments from Table I are used. The analysis is performed for third-octave frequency bands with a center frequency that is lower than $500 \mathrm{~Hz}$, for which acoustic centering using Eq. (13) can be applied. The number of local minimum points of $J_{c}$ is calculated using the MATLAB function imregionalmin. For each band and for each instrument category from Table I, the average number of minimum points is calculated over all partials in the band and all instruments from that category.

Figure 11 shows the average number of local minimum points of $J_{c}$ for all instruments from each category at the

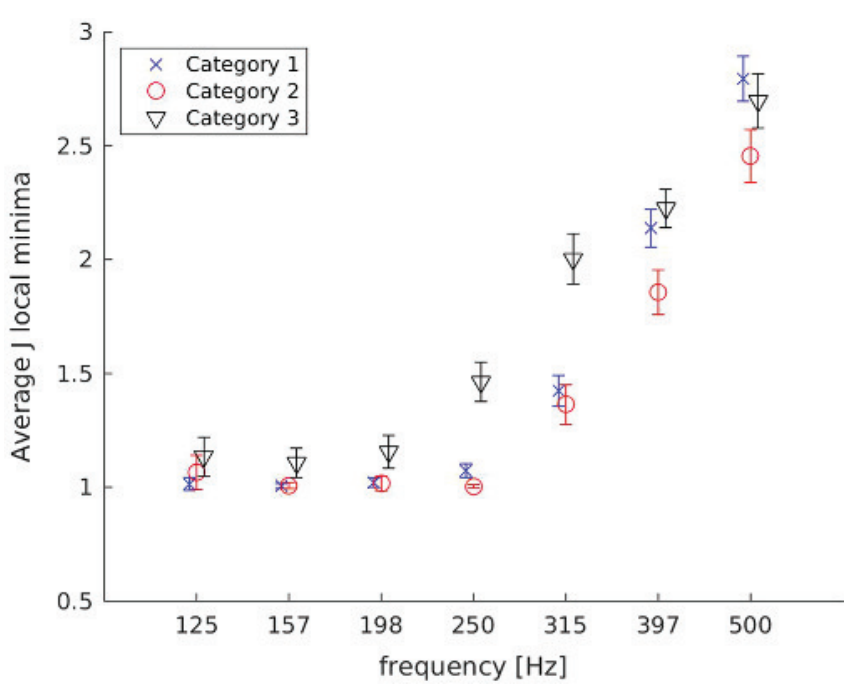

FIG. 11. (Color online) Average number of local minimum of $J_{c}$ at different frequency bands calculated for instruments at different categories. Vertical bars display $95 \%$ confidence interval. 
different demonstrated frequency bands. The vertical bars mark the $95 \%$ confidence interval. It may be seen from Fig. 11 that at all frequency bands with a center frequency below $500 \mathrm{~Hz}$, the maximum predicted number of excitation points is obtained for the musical instruments from the third category. The difference between category 1 and 2 is not significant however, as can be seen from the overlap of the $95 \%$ confidence interval. Interestingly, at the frequency band with a center frequency of $397 \mathrm{~Hz}$, instruments from category 2 show less excitation points than instruments from category 1 , and the difference between category 1 and 3 is nonsignificant. The reason might be that this frequency band contains partials at frequencies for which $J_{c}$ is not applicable anymore. Expectedly, at the frequency band with center frequency of $500 \mathrm{~Hz}$, the acoustic source objective function of $J_{c}$ does not converge well, ${ }^{18}$ and therefore the number of local minimum of the objective function cannot well predict the number of excitation points at this frequency.

\section{THE EFFECT OF A HUMAN PLAYER ON THE RADIATION PATTERN}

In this section, the radiation pattern of the violin from the generated database of this work is briefly compared to a high spatial resolution radiation pattern that was recently

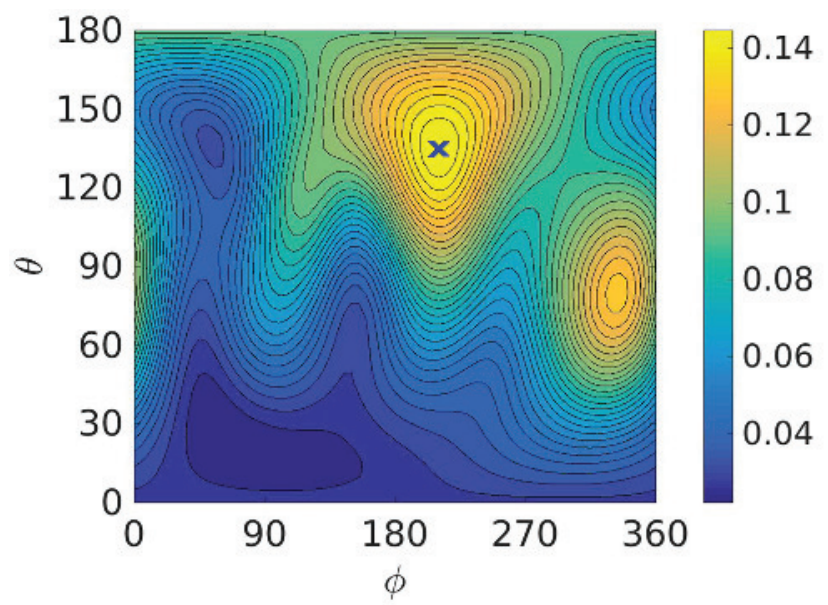

(a)

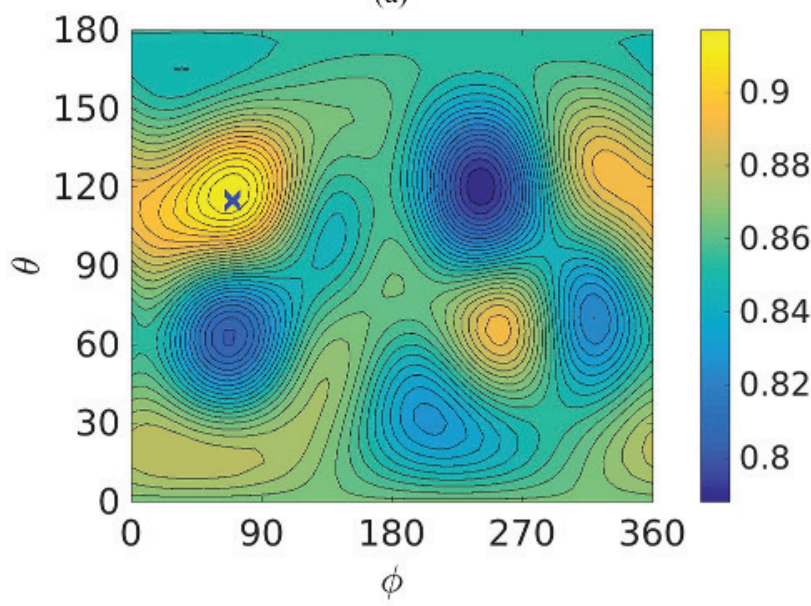

(c) recorded from a rotated and tilted electrically excited violin using a single microphone in an anechoic chamber at RWTH-Aachen University. ${ }^{17}$ The electrical excitation of the violin was obtained using an injection of a sweep current signal through the $\mathrm{G}$ string of the violin under a constant magnetic field, so that a Lorenz force vibrates the string on alternating frequency, allowing the measurement of the frequency response. The violin was rotated and tilted, and the procedure has been repeated at each position of the violin. For further details of the radiation pattern measurement of the electrically excited violin and the applied acoustic source centering in the circular harmonics domain, the reader is referred to the work of Shabtai et al. ${ }^{17}$ Thus, a graphical comparison of the radiation pattern can be performed at discrete frequencies, for the cases where a human player was present or not. A more elaborated analysis of the human player effect on the radiation pattern of more musical instruments is outside the scope of this work and subject to a future work.

Figure 12 shows the radiation pattern of the violin with and without the player at the partial frequencies of 197 and $590 \mathrm{~Hz}$. The first clear difference between the radiation patterns at both frequencies can be seen by comparing Figs. 12(a) and 12(b) to Figs. 12(c) and 12(d), respectively. The

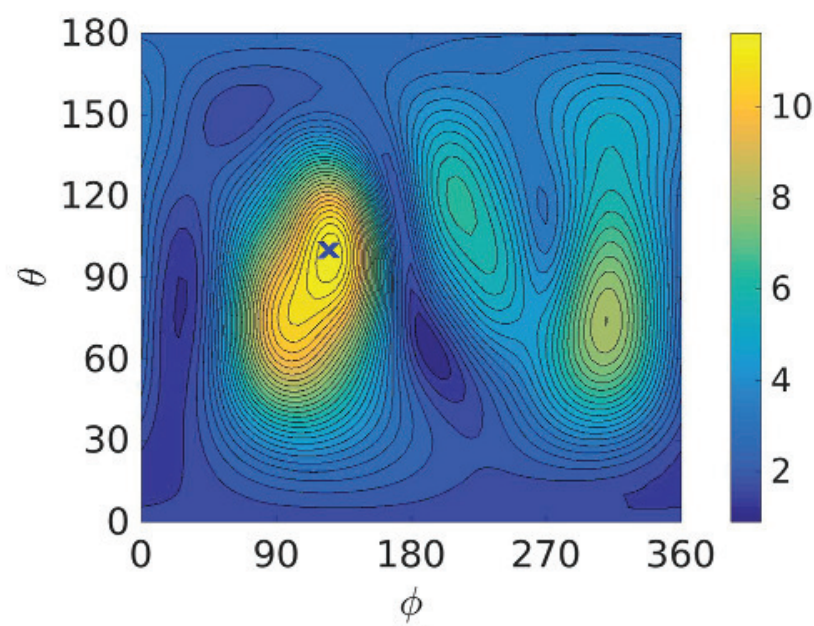

(b)

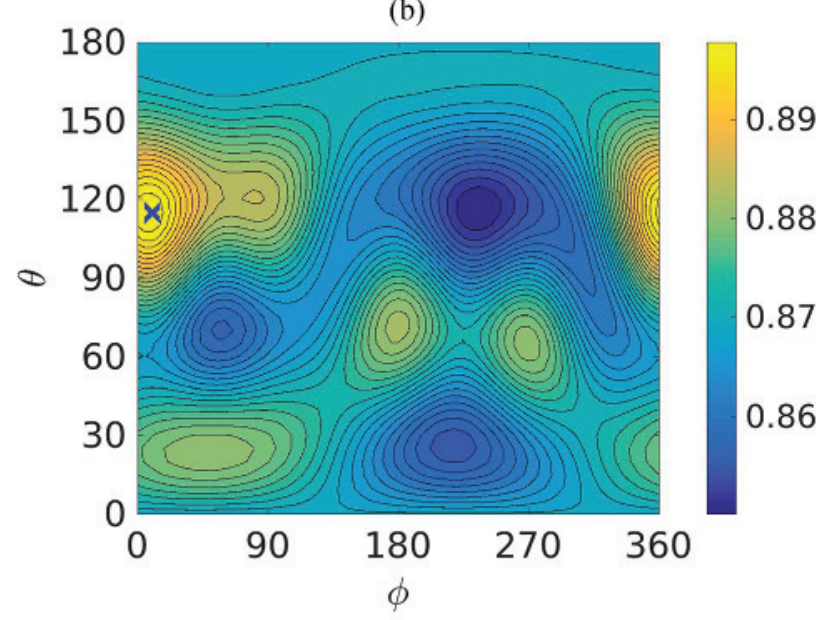

(d)

FIG. 12. (Color online) Radiation patterns of the violin with a human player at (a) $197 \mathrm{~Hz}$ and (b) $590 \mathrm{~Hz}$, and of the electrically excited violin at (c) $197 \mathrm{~Hz}$ and (d) $590 \mathrm{~Hz}$. 
radiation pattern of the recorded signal with the player present [Figs. 12(a) and 12(b)] shows more directions to which a lobe of acoustic pressure is radiated with an energy peak, than is shown in the radiation pattern recorded without the player [Figs. 12(c) and 12(d)]. This result implies that the body of the player may smooth the directionality of the radiation pattern. Further differences can be noted by the location of the main lobes, but these may be relevant to the initial positioning of the violin at the $(0,0)$ angle rather than the presence of the human player, because the change in direction of the main lobe due to the change in frequency is persistent between the cases in which a player was present or not.

\section{CONCLUSION}

The spatial sound of 41 musical instruments was recorded using a surrounding spherical array of 32 microphones in an anechoic chamber. Acoustic source centering is applied to all instruments, but to frequencies that are lower than $1 \mathrm{kHz}$. The database is stored in a compact manner in the spherical harmonics domain of order 4 , resulting in 25 coefficients for each third-octave band. An analysis of the acoustic source centering has been performed assuming that the musical instruments may be divided into different categories with respect to the number of sources that generate the acoustic radiation pattern. This database can be used in studies related to musical sound radiation in general, and in virtual-acoustics computer software. The database is available on "Deposit Once, Repository for Research Data and Publications," under https://www.depositonce.tu-berlin.de. ${ }^{25}$ The mat files in the database contain the $p_{n m}$ values at a distance of $2.1 \mathrm{~m}$ at each third-octave frequency band, where centered radiation patterns of partials under $1 \mathrm{kHz}$ and noncentered radiation patterns of partials over $1 \mathrm{kHz}$ were averaged.

${ }^{1}$ J. B. Allen and D. A. Berkley, "Image method for efficiently simulating small-room acoustics," J. Acoust. Soc. Am. 65, 943-950 (1979).

${ }^{2} \mathrm{P}$. Peterson, "Simulating the response of multiple microphones to a single acoustic source in a reverberant room," J. Acoust. Soc. Am. 80, $1527-1529$ (1986).

${ }^{3}$ D. S. Brungart and W. M. Rabinowitz, "Auditory localization of nearby sources. Head-related transfer functions," J. Acoust. Soc. Am. 106, 1465-1479 (1999).

${ }^{4}$ F. Menzer, C. Faller, and H. Lissek, "Obtaining binaural room impulse responses from b-format impulse responses using frequency-dependent coherence matching," IEEE Trans. Audio, Speech, Lang. Process. 19, 396-405 (2011).

${ }^{5} \mathrm{M}$. Vorländer, "Simulation of the transient and steady-state sound propagation in rooms using a new combined ray-tracing image-source algorithm," J. Acoust. Soc. Am. 86, 172-178 (1989).
${ }^{6}$ D. Schröder and M. Vorländer, "Raven: A real-time framework for the auralization of interactive virtual environments," in Forum Acusticum (Aalborg, Denmark, 2011), pp. 1541-1546.

${ }^{7}$ G. M. Naylor, "ODEON-Another hybrid room acoustical model," Appl. Acoust. 38, 131-143 (1993).

${ }^{8}$ M. Kompis and N. Dillier, "Simulating transfer functions in a reverberant room including source directivity and head-shadow effects," J. Acoust. Soc. Am. 93, 2779-2787 (1993).

${ }^{9} \mathrm{~J}$. Meyer, Acoustics and the Performance of Music, 5th ed. (Springer, New York, 2009), Chap. 4, pp. 129-178.

${ }^{10} \mathrm{P}$. Cook and D. Trueman, "A database of measured musical instrument body radiation impulse responses, and computer applications for exploring and utilizing the measured filter functions," in Proceedings of the 23rd International Symposium on Musical Acoustics (ISMA 23), Woodbury, New York (1998), pp. 303-308.

${ }^{11}$ F. Otondo and J. H. Rindel, "The influence of the directivity of musical instruments in a room," Acta Acust. Acust. 90, 1178-1184 (2004).

${ }^{12}$ B. F. G. Katz and C. d'Alessandro, "Directivity measurements of the singing voice," in Proceedings of the 19th International Congress on Acoustics (ICA 2007), Madrid, Spain (2007).

${ }^{13} \mathrm{~F}$. Hohl, "Kugelmikrofonarray zur Abstrahlungsvermessung von Musikinstrumenten (Spherical microphone array for capturing soundradiation from musical instruments)," Master's thesis, Institute of Electronic Music and Acoustics, University of Music and Performing Arts, Graz, Austria (2009).

${ }^{14}$ J. Pätynen and T. Lokki, "Directivities of symphony orchestra instruments," Acta Acust. Acust. 96, 138-167 (2010).

${ }^{15}$ M. Pollow, G. K. Behler, and B. Masiero, "Measuring directivities of natural sound sources with a spherical microphone array," in Proceedings of the Ambisonics Symposium, Graz, Austria (2009), pp. 166-169.

${ }^{16}$ T. Grothe and M. Kob, "Investigation of bassoon directivity," in Proceedings of the Stockholm Music Acoustics Conference (SMAC), Stockholm, Sweden (2013), pp. 391-397.

${ }^{17}$ N. R. Shabtai, G. Behler, and M. Vorländer, "Generation of a reference radiation pattern of string instruments using automatic excitation and acoustic centering," J. Acoust. Soc. Am. 138, EL480-EL486 (2015).

${ }^{18}$ I. Ben Hagai, M. Pollow, M. Vorländer, and B. Rafaely, "Acoustic centering of sources measured by surrounding spherical microphone arrays," J. Acoust. Soc. Am. 130, 2003-2015 (2011).

${ }^{19}$ N. R. Shabtai and M. Vorländer, "Acoustic centering of sources with high-order radiation patterns," J. Acoust. Soc. Am. 137, 1947-1961 (2015).

${ }^{20}$ G. K. Behler, M. Pollow, and M. Vorländer, "Measurements of musical instruments with surrounding spherical arrays," in Proceedings Acoustics, Nantes, France (2012), pp. 755-759.

${ }^{21}$ M. Pollow, "Directivity patterns for room acoustical measurements and simulations," Ph.D. thesis, Institute of Technical Acoustics, RWTH Aachen University, Aachen, Germany (2015).

${ }^{22}$ ISO 16:1975, "Acoustics-Standard tuning frequency (standard musical pitch)" (International Organization for Standardization, Geneva, Switzerland, 1975).

${ }^{23}$ E. G. Williams, Fourier Acoustics: Sound Radiation and Nearfield Acoustical Holography (Academic, New York, 1999), Chap. 6, pp. $183-234$.

${ }^{24} \mathrm{~F}$. Wefers, "A free, open-source software package for directional audio data," in Proceedings of the 36th German Annual Conference on Acoustics (DAGA 2010), Berlin, Germany (2010).

25“Deposit Once, Repository for Research Data and Publications," https:// www.depositonce.tu-berlin.de (Last viewed 2/23/2017). 